\title{
Elman Recurrent Neural Network Application in Adaptive Beamforming of Smart Antenna System
}

\author{
Adheed H. Sallomi, PhD \\ Assist Prof., \\ Electrical Engineering Department, \\ Al-Mustansiryah University,Baghdad, Iraq
}

\author{
Sulaiman Ahmed \\ Electrical Engineering Department, \\ Al-Mustansiryah University, \\ Baghdad, Iraq
}

\begin{abstract}
In this paper an artificial Elman Recurrent Neural Network (ERNN) is used for smart antenna adaptive beamforming. Neural network is used to calculate the optimum weights of uniform linear array antenna that steer the radiation pattern of the antenna by directing multiple narrow beams toward the desired users and make nulling in the direction of unwanted users. Two different supervised training algorithms are used to train the ERNN, they are Levenberg Marquardt (LM) algorithm and Resilient Backpropagation (Rprop) algorithm. Uniform linear array is used with five element and the spacing between element equal to half wavelength. The results of ERNN training using LM and Rprop showed that the performance of Neural Network (NN) trained by LM training algorithm is better than Rprop training algorithm , since it consider the fastest backpropagation training algorithm but it requires more memory than other algorithms.
\end{abstract}

\section{Keywords}

Smart Antenna, Conventional and Adaptive Beamforming, Elman Recurrent Neural Network.

\section{INTRODUCTION}

In modern mobile communication system, the demands for best coverage, higher quality of transmission and capacity improvement are constantly growing. The using of omnidirectional or sectored antennas in a conventional cellular system to make connection between the mobile user and the base station lead to waste a lot of power as the radiated power doesn't focus in specific directions [1,2]. The mobile communication system is influenced by three main impairments, which are: co-channel interference, delay spread and multipath[3].Smart antenna systems introduce an effective solution to the present impairments of wireless systems and achieving high speed and high data rate of transmission. Smart antenna system can increase system capacity by focusing the maximum radiation toward desired users, while nulling interference sources. This allows higher frequency reuse within the same cell ,higher signal to interference (SIR) ratios and lower power consumption[4,5]. The adaptive beamforming algorithms that used by signal processing unit of smart antenna system give the ability of smart antenna to enhance the performance of mobile communication system [6,7].This paper introduces proposed model of smart antenna adaptive beamforming based on ERNN. The neural network trained to calculate the optimum weights in order to direct narrow beam patterns of the arrays to the desired directions of targets and make nulling toward the direction of interfering sources. Neural network methods for smart antenna adaptive beamforming introduces such advantages such as, ,fast convergence rates, increase system capacity, nonlinear property and adaptive learning capability.

\section{SMART ANTENNA SYSTEM}

Smart antenna system is an array of antennas that terminated into an intelligence signal processing unit to make the transmission and reception of antenna in an adaptive, spatially sensitive manner .Smart antennas have the ability of separating signals from numerous sources can substantially enhance the performance of cellular communications systems [8]. The ability and the Intelligence of smart antenna systems is come from the adaptive algorithms that used by the digital signal processing (DSP) unit of antenna array system . the block diagram of smart antenna system with $M$ elements is shown in Fig.1 [9,10,11].

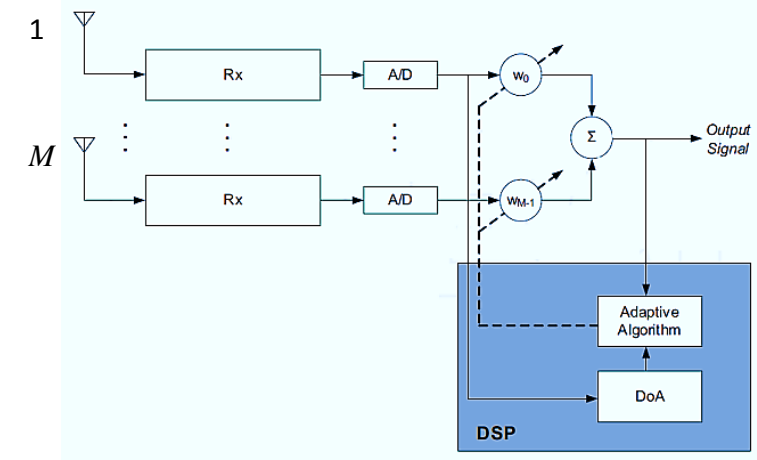

Fig1: Block diagram of smart antenna system

Where, $\mathrm{A} / \mathrm{D}$ refer to analog to digital convertor, $\mathrm{W}_{0} \ldots . . \mathrm{w}_{\mathrm{M}-1}$ is the weights of antenna array that calculated by the adaptive algorithm of DSP unit to steer maximum radiation of antenna array toward the desired users and nulling interference users. DOA refer to direction of arrival estimator that estimate the angle of arrival of the incoming signals. There are two main type of smart antenna beamforming, which are conventional beamforming and adaptive beamforming.

\section{CONVENTIONAL BEAMFORMING}

In a conventional beamforming smart antenna use fixed predefined weights to study the signal arriving from a specific direction. Since it enhance the signal incoming from desired direction while nulling signals from other directions, thus it is called the spatial matched filter[12].In the fixed weight beamforming it assumed the angle of arrival of received signals does not change with time, so the optimum weight would not need to be adjusted[2].Some of fixed weights beamforming algorithm that used by antenna array such as: Minimum Mean-Square Error Method, Maximum Signal-toInterference Ratio, Minimum Variance (MV) Method and Maximum Likelihood Method. Block diagram of fixed weight beamformer is shown in Fig.2[13]. 


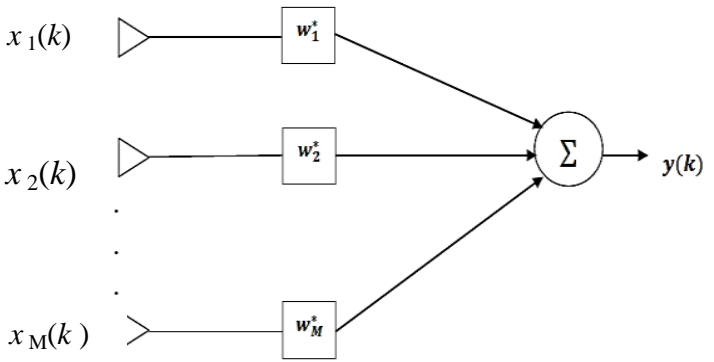

Fig 2:Fixed weight beamformer

the weighted array output of antenna array can be given in the following form[8]:

$(k)=w^{H} \cdot x(k)$

where:

$$
\begin{aligned}
(k)= & a_{0} s(k)+\left[a_{1} a_{2} \ldots . . a_{N}\right] \cdot\left[i_{1}(k) i_{2}(k) . . i(k)\right]^{\mathrm{T}}+\mathrm{n}(k) \\
& =x(k)+x_{i}(k)+n(k)
\end{aligned}
$$

$w=\left[w_{1} w_{2} \ldots w_{M}\right]^{T}=$ weights of Array

$x(k)=$ desired vector of signal

$x(k)=$ vector of interfering signals

$(k)=$ zero mean Gaussian noise for each channel

$a_{i}=$ steering vector of $M$-element array for $\theta_{i}$ direction of arrival

\section{ADAPTIVE BEAMFORMING}

Adaptive Beamforming is a signal processing technique in which the received signals by each element of antenna array are multiplied by complex weight vectors to adapt the magnitude and phase of the received signals in order to direct narrow beam patterns in the direction of desired users and nulling toward the interferer sources. The signals received by different elements of an antenna array combined to form a single output. Classically, this is achieved by decreasing the mean square error (MSE) between the actual array output and desired output [14].Fig.3 shows the block diagram of adaptive beamformer.

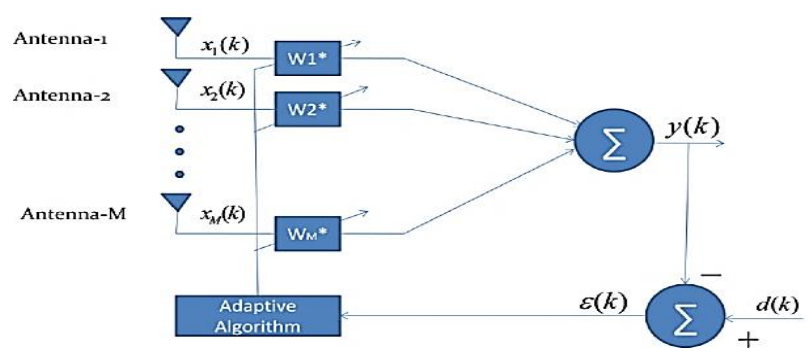

Fig 3: Adaptive beamformer

\section{ARTIFICIAL NEURAL NETWORK (ANN)}

ANN is an applications of artificial intelligence that attempt to simulate the human brain activities in its functionalities and structure in a mathematical model. In artificial neural nets the "artificial" is included to distinguish the computer-based systems from the biological neural network system. ANNs have become very common in a broad domains including, medical ,industrial and financial applications[15]. Also neural networks are widely used in the field of signal processing mainly because of their, fast convergence rates, general purpose nature ,and its ability to store the experimental knowledge and making it available for use[16]. There are three features to characterize a neural network is:

1. Architecture: the connection pattern of nodes between neurons.

2. The learning or training algorithm : the method of the weights calculations on the connections.

3. The activation function: the function that achieve an output for the input values received by a node [17].

\section{RECURRENT NEURAL NETWORK (RNN)}

Recurrent network is a special type of artificial neural network. RNN consist of either single layer or multiple hidden layers network. They have one or more feedback loops and this consider the basic difference from feed forward networks as shown in Fig.4 .The feedback loop can appear between any two neurons or layers in many forms. It typically contain unit delay element denoted by $z^{-1}$. Because recurrent networks consist of a large number of feed forward and feedback connections, they exhibit complex dynamics. Recurrent networks used in wide applications as system identification, intelligent control and applications of dynamical system[17].The unit delay element of the feed- back loops indicates that the RNN has local memory characteristics have the ability to store activity patterns and introduce them to the neural network more than once, therefore the layers with feedback connections can use their past activation in them previous behavior. Therefore, at any given time, the output of network can be calculated by propagating the input pattern forward through the $\mathrm{NN}$ and the recurrent activations are propagated backward to the additional layer that known as context layer ,that able to copy the activation pattern from the output layer on the last instant. The most common recurrent neural networks were produced by Jordan and Elman where Jordan is fully recurrent neural network (all existing neurons output are used for feedback) and ELMAN is partially recurrent neural network [18, 19, 20].

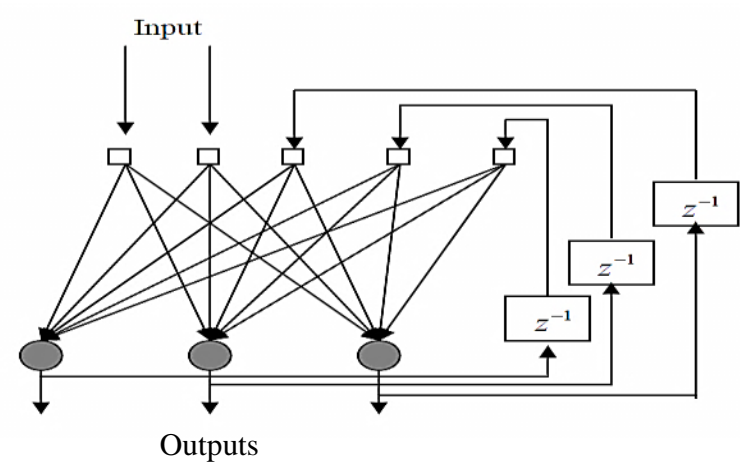

Fig 4: Recurrent neural network

\section{MATHEMATICAL MODEL OF UNIFORM LINEAR ARRAY}

It is assumed that there are $K$ narrow-band incoherent plane waves, received by a Uniform Linear Array (ULA) with $M(M>K)$ omnidirectional antenna elements spaced by distance $d$ from directions $\left\{\theta_{1}, \theta_{2}, \ldots . \theta_{K-1}\right\}$,which are between [-90,90].the geometry of linear array antenna is shown in Fig.5. 


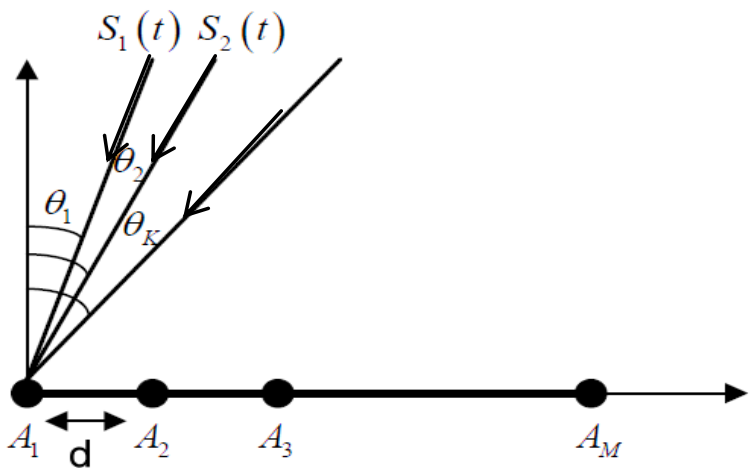

Fig 5:Geometry of linear array antenna

the received signal at $M_{\text {th }}$ antenna element is calculated by [11]:

$X_{i}(k)=\sum_{m=1}^{K} s_{m}(k) e^{-j(i-1) k_{m}}+n_{i}(k)$

$\mathrm{i}=1,2, \ldots \ldots . M$

where $: \mathrm{s}_{m}$ are the steering vector of signals received from $\mathrm{i}^{\text {th }}$ sensor, $n_{i}(t)$ is the noise received at each element of array antenna and

$k_{m}=\frac{\omega_{0} d}{c} \sin \left(\theta_{m}\right)$

(4)

where: $d$, is the distance between the array elements, $\omega_{0}$ is the angular frequency and c refer to the light speed in free space.

The formula of the received signal can be written in matrix form as :

$X(k)=\mathrm{A} S(k)+N(k)$

Where:

A is array steering matrix toward the direction of the incoming signal and is given by:

$$
\begin{aligned}
& \mathrm{A}=\left[\mathrm{a}\left(\theta_{1}\right), \mathrm{a}\left(\theta_{2}\right) \ldots \ldots \mathrm{a}\left(\theta_{\mathrm{k}}\right)\right] \\
& \mathrm{a}\left(\theta_{\mathrm{m}}\right)=\left[\begin{array}{lll}
1 & e^{-j k_{m}} & e^{-j 2 k_{m}} \ldots \ldots e^{-j(M-1) k_{m}}
\end{array}\right] \\
& X(k)=\left[x_{1}(k) x_{2}(k) \ldots \ldots . . x_{m}(k)\right]^{\mathrm{T}} \\
& N(k)=\left[\begin{array}{lllll}
n_{1}(k) & n_{2}(k) & \ldots \ldots & n_{m}(k)
\end{array}\right]^{\mathrm{T}} \\
& S(k)=\left[s_{1}(k) s_{2}(k) \ldots \ldots \ldots s_{m}(k)\right]^{\mathrm{T}}
\end{aligned}
$$

array output $y$ can be given as follows:

$y(k)=w^{H} \cdot X(k)$

where:

$w=\left[\begin{array}{llll}w_{1} & w_{2} & \ldots & w_{M}\end{array}\right]^{T}$

$w$ :weight vector of antenna array.

\section{BEAMFORMING PROCEDURE}

There are two steps of using neural network for smart antenna beamforming :

\subsection{Training phase}

In this step the input and output pairs of artificial neural network are generated(preprocessing) in order to train the network on them and saving the weights after achieving best training performance. At first the correlation matrix of the incoming signals that received by $M$ elements of antenna array system are generated as follows:

$\mathrm{R}_{\mathrm{xx}}=\mathrm{E}\left\{\mathrm{X}(\mathrm{k}) \mathrm{X}(\mathrm{k})^{\mathrm{H}}\right\}=\left[\begin{array}{cccc}R_{11} & R_{12} & \ldots \ldots & R_{1 M} \\ R_{21} & R_{22} & \ldots \ldots & R_{2 M} \\ \cdot & \cdot & . & \cdot \\ \cdot & \cdot & \cdot \\ R_{M 1} & R_{M 2} & \ldots \ldots & R_{M M}\end{array}\right]$

where $\mathrm{H}$ refer to conjugate transpose, then the first row of the correlation matrix $\mathrm{R}_{\mathrm{xx}}$ is taken to be as the input of neural network since it contain adequate information about the received signal as:

$Z=\left[\begin{array}{llll}R_{11} & R_{12} & \ldots \ldots \ldots . . . R_{1 M}\end{array}\right]$, then the input vector is normalized to be more suitable as input of neural network :

$$
B=\frac{Z}{\|Z\|}
$$

and because the neural network does not operate with complex number, therefore the real and imaginary part of each element in $B$ vector is taken, so the dimension of $B$ vector will be twice $(1 \times 2 M)$.The target output of neural network is generated from the weight equation of Minimum Variance Distortionless (MVDL) beamformer. The weights that generated from MVDL beamformer can provide optimal beamforming and steer the main beam of radiation pattern toward desired users and nulling undesired users in optimal form. The antenna array weight based on MVDL beamformer is given in eq.(15)[21].

$$
W_{o p}=\frac{A_{d} R_{x x}^{-1}}{A_{d}^{H} R_{x x} A_{d}^{-1}}
$$

Where $A_{d}$ refer to the steering vector of $K$ desired signals received by antenna array with $M$ elements. The neural network training process is shown in Fig.6.

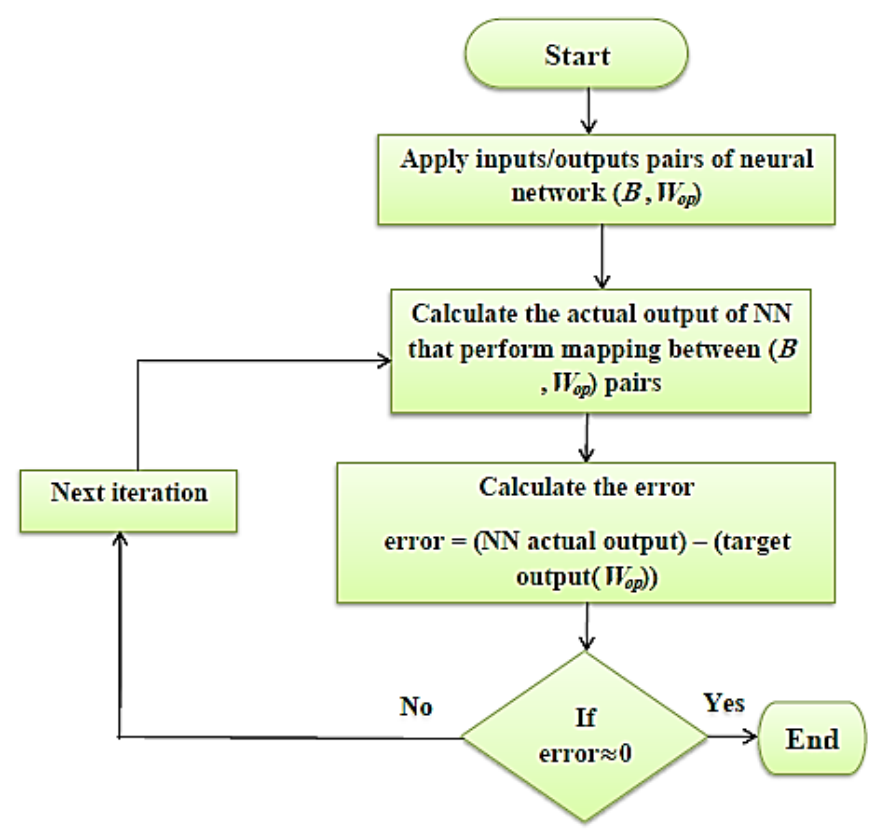

Fig 6:Training process of neural network

As shown in Fig.6 the training is complete after the network reach to the best training performance ( the error between actual and target output $\left(W_{o p}\right)$ approach to zero ) ,then the network weights are saved. Where, the network will use these weights to estimate the output of data sample that network did not trained on them before (unseen data sample ). It is worth mentioning that the network needs more than one trial of training to reach to best training performance and the saved 
weights of the network refer to empirical knowledge that gained by the network during the training process.

\subsection{Performance Phase}

In this step the neural network will estimate the weights of the new incoming signals that network never seen before during training step (network did not trained on them) ,the correlation matrix of the received signal are calculated ,and then the only input to the neural network is $B$ vector that given in eq(14), and the network will estimate (or predict) the output (optimum weight $\left(W_{o p}\right)$ ) based on empirical knowledge gained through the training process. These weights are used by antenna array system to form multiple narrow beams toward the desired directions and make nulling in the direction of interferences.

The proposed model of ERNN is shown in Fig.7, it consists of three layers with context unit, both input and output layer consist of 3 nodes (equal to the number of training data sample), while the hidden layer consist of five nodes (based on trial and error). The activation functions that used by ERNN in both hidden and output layer is a tan sigmoid transfer functions. ERNN is trained by using supervised training algorithms, they are : Levenberg Marquardt (LM) algorithm and Resilient backpropagation(Rprop) algorithm.

Elman Recurrent Neural Network (ERNN) model is shown below:

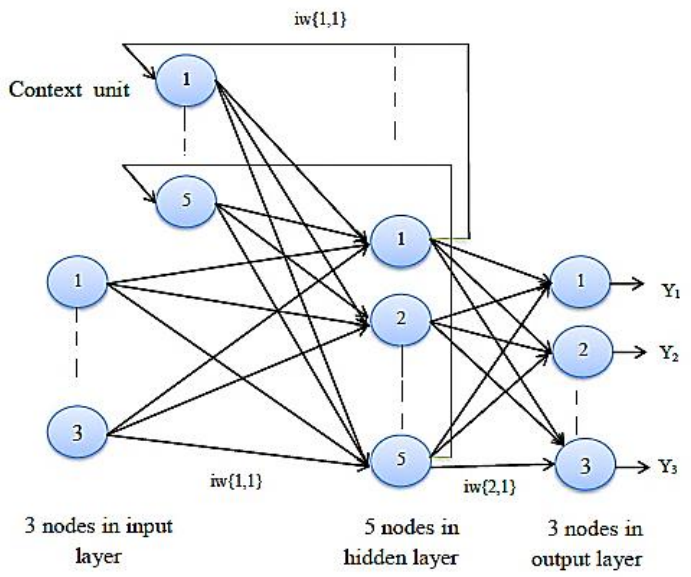

Fig 7 : EIman Recurrent neural network

where : $\operatorname{iw}\{1,1\}$ is the weights matrix between the hidden layer and the context unit, $\operatorname{iw}\{2,1\}$ is the weight matrix between the output layer and the hidden layer, $\mathrm{Y}_{1}, \mathrm{Y}_{2}$ and $\mathrm{Y}_{3}$ refer to the outputs of ERNN. Also ,the input/output pairs $(B$ , $\left.\mathrm{W}_{\mathrm{op}}\right)$ are generated in order to train the ERNN on desired weight vectors. It is found that the network is able to estimate the weights of all unseen signals between the range $\left[-90^{\circ}, 90^{\circ}\right]$ if the ERNN trained on the signals that received from the following direction of arrival: $\left[-90^{\circ}, 0^{\circ}, 90\right]$. The weights vectors (iw $\{1,1\}$ and $\operatorname{iw}\{2,1\}$ ) are saved after reaching to best training performance and the network tested for new unknown received signals, the only input to the network is $B$ vector and the output will be the optimum weight $\left(\mathrm{W}_{\mathrm{op}}\right)$.After achieving the output from the artificial neural network training, the beam pattern of antenna array system is drown from the equation of array factor that given as follows :

$A F=\left|W_{o p}(k) e^{-j(i-1) \alpha(\mathrm{k})}\right|$ where, $\alpha(\mathrm{k})$ is the searching angle between $\left[-90^{\circ}, 90^{\circ}\right]$ with $1^{\circ}$ as step size from $-90^{\circ}$ to $90^{\circ}$.

\section{SIMULATION RESULTS}

The simulation results of smart antenna beamforming based on ERNN are implemented for uniform linear array with $(M=5)$ and element spacing $d=\lambda / 2$. ERNN has been trained using the following supervised algorithms:

\subsection{Levenberg-Marquardt (Im) Training}

The performance of ERNN training phase based on Levenberg-Marquardt (LM) algorithm, with five hidden neurons is shown in Fig. 8 ,where the best phase training performance is [1.121938e-14] at epoch 38 with best validation performance equal to [1.682442e-14] and the best test performance is [3.363946e-14].

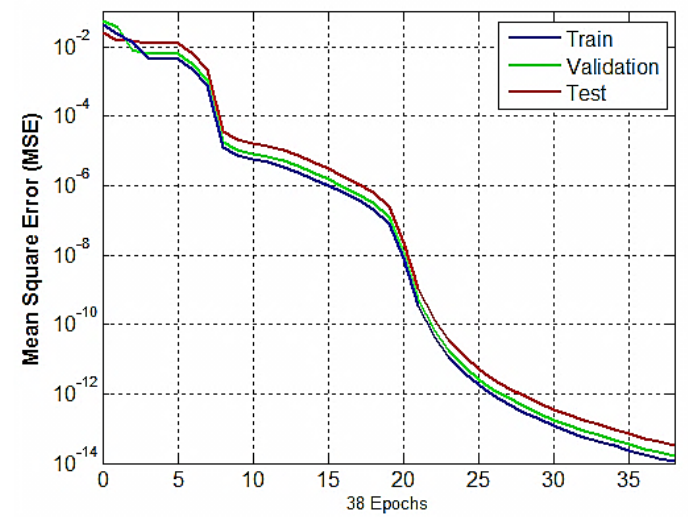

Fig 8: Performance of ERNN trained by LM algorithm

After the ERNN training phase based on LM algorithm is completed, the network had been tested for some new unseen signals and has given good performance .Fig.9 shows the linear and polar plot of beam pattern (uniform Array factor (AF) with respect to Angle Of Arrival (AOA)) of new incoming signal with DOA $=30^{\circ}$, As shown in Fig.9 the main beam of antenna based on ERNN almost completely identical with the actual main beam(based on MVDL beamformer). The side lobe level of antenna beam pattern based on ERNN trained by LM algorithm is about 0.05 above the actual value.
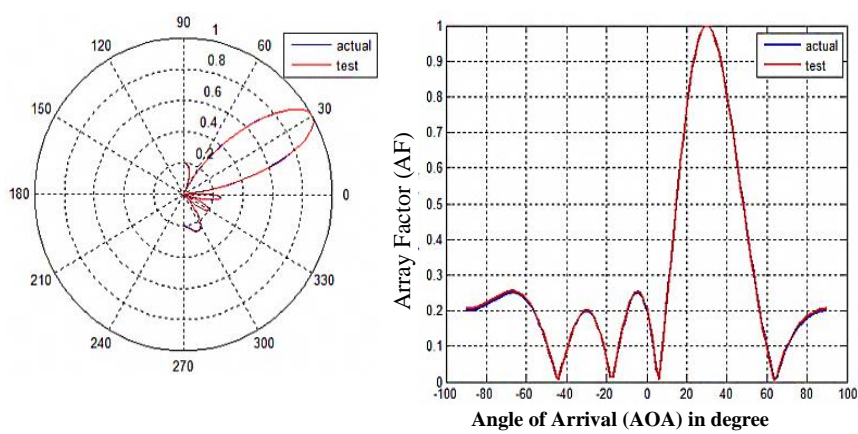

Fig 9 : Antenna beam pattern using ERNN trained by $\mathrm{LM}$ algorithm $\left(M=5, \mathrm{~d}=\lambda / 2, \mathrm{DOA}=30^{\circ}\right)$

Fig. 10 shows the linear and polar plot of new incoming signal with $\mathrm{DOA}=60^{\circ}$, the main beam identical with actual one and the side lobe level increased by 0.18 above the side lobe of actual beam.

$i=1,2 \ldots . . M$ 

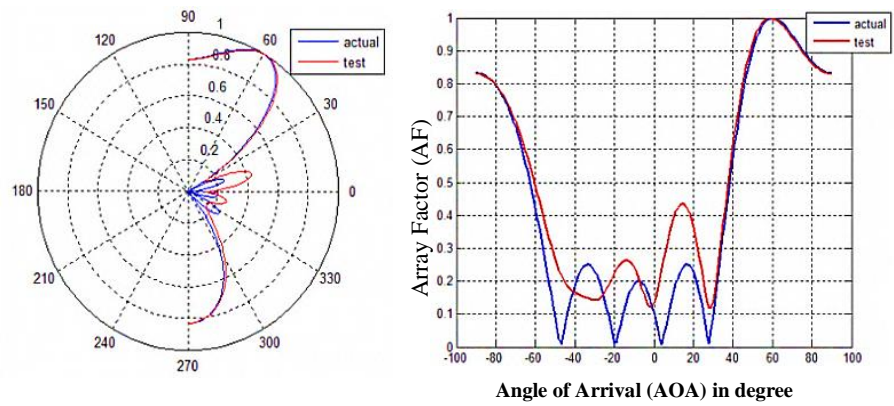

Fig 10 : Antenna beam pattern using ERNN trained by $\mathrm{LM}$ algorithm $\left(M=5, \mathrm{~d}=\lambda / 2, \mathrm{DOA}=60^{\circ}\right)$

\subsection{Resilient Back-Propagation (Rprop) Training}

The performance of ERNN training that updates weight and bias values based on the Rprop training algorithm with five hidden neurons is shown in Fig. 11 , where the best phase training performance is [1.358237e-07]at epoch 79 with best validation performance is [3.494906e-07] and best test performance is [3.704479e-07].

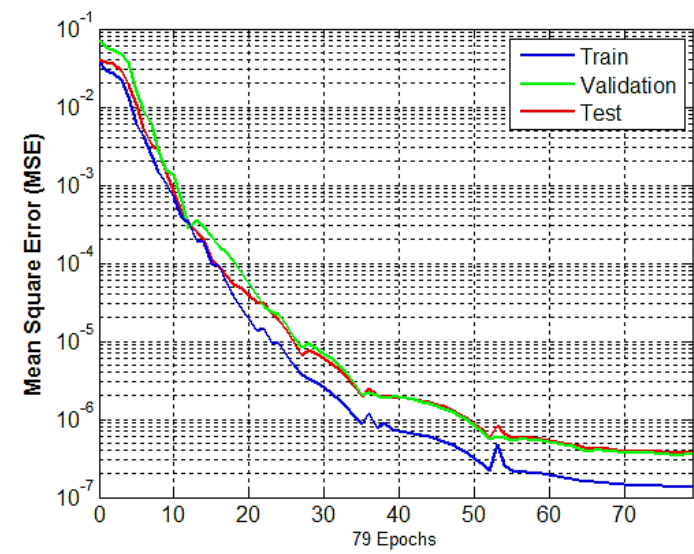

Fig 11: Performance of ERNN trained by Rprop algorithm

After the ERNN training phase based on Rprop algorithm is completed, the network had been tested for some new unseen signals and has given good performance. Fig.12 shows the linear and polar plot of beam pattern of new incoming signal with $\mathrm{DOA}=45^{\circ}$, As shown in Fig. 12 the main beam of antenna based on ERNN almost completely identical with the actual main beam. The side lobe level of antenna beam pattern based on ERNN trained by Rprop algorithm is about 0.12 above the actual value.
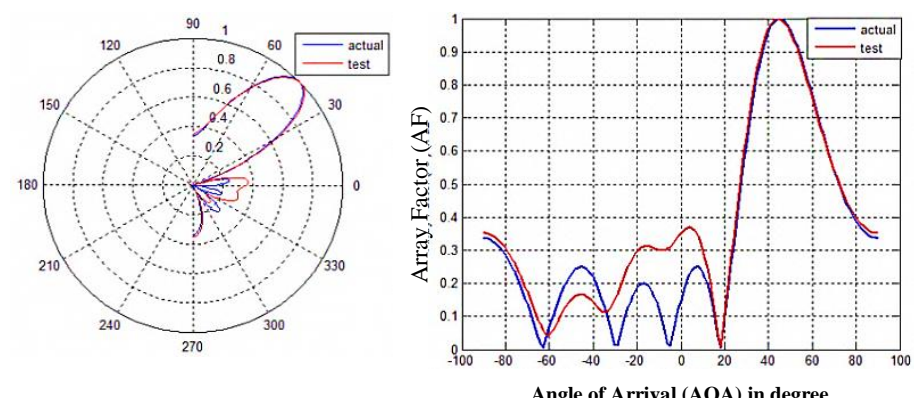

Fig 12 : Antenna beam pattern using ERNN trained by Rprop algorithm $\left(M=5, d=\lambda / 2, \mathrm{DOA}=45^{\circ}\right)$
Fig.13 shows the linear and polar plot of new incoming signal with $\mathrm{DOA}=-10^{\circ}$, the main beam identical with actual one and the side lobe level increased by 0.05 above the side lobe of actual beam.
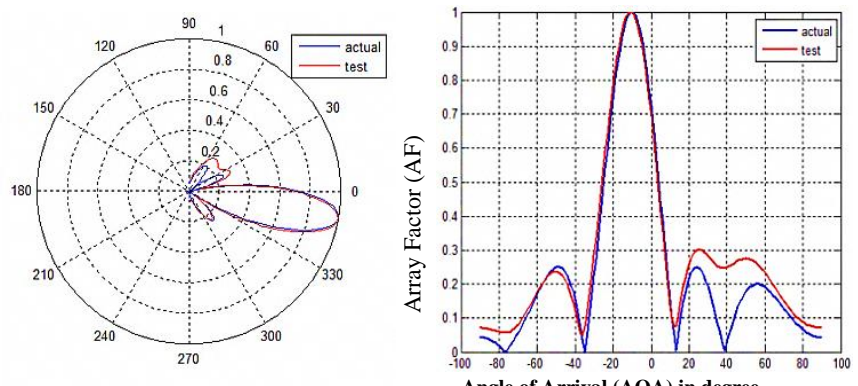

Fig 13 : Antenna beam pattern using ERNN trained by Rprop algorithm $\left(M=5, d=\lambda / 2, \mathrm{DOA}=-10^{\circ}\right)$

Table(1) shows the comparison between the performance of ERNN trained by LM and Rprop, where LM has given better performance than Rprop training algorithm with less number of epoch but it requires more memory than other algorithms.

Table 1 : Comparison between the performance of ERNN trained by LM and Rprop

\begin{tabular}{|c|c|c|}
\hline Performance/Training algorithm & LM & Rprop \\
\hline Training performance & $1.121938 \mathrm{e}-14$ & $1.358237 \mathrm{e}-07$ \\
\hline Validation performance & $1.682442 \mathrm{e}-14$ & $3.494906 \mathrm{e}-07$ \\
\hline Test performance & $3.363946 \mathrm{e}-14$ & $3.704479 \mathrm{e}-07$ \\
\hline epoch & 35 & 79 \\
\hline
\end{tabular}

Table (2) shows the amount of the increase in the side lobe level of antenna array beam pattern based on ERNN from the actual value for 10 signals. The results show that the amount of increase in the side lobe level is almost equal for both LM and Rprop, but it may be differs from one angle of arrival to another, because the inputs/outputs pairs of neural network $(B$ ,$\left.W_{o p}\right)$ may contain approximately equal neighboring samples , and it has been proven by experience this will adversely affect the performance of the neural network.

Table 2 : The amount of in the side lobe level in the beam pattern of antenna array pattern based on ERNN from the actual value for 10 signals.

\begin{tabular}{|c|c|c|}
\hline DOA / Training algorithm & LM & Rprop \\
\hline $30^{\circ}$ & 0.05 & 0.01 \\
\hline $60^{\circ}$ & 0.18 & 0.16 \\
\hline $45^{\circ}$ & 0.12 & 0.12 \\
\hline$-10^{\circ}$ & 0.05 & 0.05 \\
\hline$-20^{\circ}$ & 0.133 & 0.133 \\
\hline $40^{\circ}$ & 0.18 & 0.18 \\
\hline $10^{\circ}$ & 0.05 & 0.05 \\
\hline$-30^{\circ}$ & 0.01 & 0.01 \\
\hline$-60^{\circ}$ & 0.15 & 0.15 \\
\hline$-40^{\circ}$ & 0.18 & 0.18 \\
\hline
\end{tabular}

\section{CONCLUSION}

In this paper Elman Recurrent Neural Network (ERNN) is used for smart antenna adaptive beamforming. ERNN is trained using LM and Rprop back propagation training algorithm. ERNN is trained to calculate the optimum weights of antenna array to direct multiple narrow beam patterns toward the desired directions of users and make nulling toward the direction of interfering sources. LM has been given a better performance than Rprop training algorithm but it 
needs more memory than other algorithms .Neural network application in adaptive beamforming for smart antenna system introduces such advantages such as, fast convergence rates, nonlinear property ,increase capacity of system and adaptive learning capability. As a future extension for this method it is possible to increase the number of desired users (targets) and direct multiple narrow beams toward them and simultaneously nulling interference sources, as well as using lower complexity ways in the hybridization process between the artificial neural network and antenna array.

\section{REFERENCES}

[1] RK Jain, Sumit Katiyar and NK Agrawal, "Smart Antenna for Cellular Mobile Communication" VSRD International Journal of Electrical, Electronics \& Communication, Department of Electrical \& Electronics Engineering, Singhania University, Jhunjhunu, Rajasthan, INDIA, Vol. 1 (9), 2011, pp.530-541.

[2] Hung Tuan Nguyen ,"Multiple Antenna Systems for Mobile Terminals", PhD. Thesis , Department of Communication Technology., Aalborg University, Denmark, 2005.

[3] Jack H.Winters AT and T Labs, "Smart Antennas For Wireless Systems" IEEE Personal Communication, ISSN :1070-9916 February 1998,pp.23-27.

[4] Frank Gross, "Smart Antenna for Wireless Communications “, Mcgraw-hill, September, 2005.

[5] Murray, B.P. and Zaghloul, A.L. "Survey Of Cognitive Beamforming Techniques " IEEE ,Dept. of Electrical and Computer Engineering, Virginia Tech, Blacksburg, VA, USA, ISBN:978-1-4799-3119-4 ,January 2014

[6] Nwalozie G.C, Umeh K.C, Okorogu V.N and Oraetue C.D, "Performance Analysis of Constant Modulus Algorithm (CMA) Blind Adaptive Algorithm for Smart Antennas in a W-CDMA Network", International Journal of Engineering Science and Innovative Technology (IJESIT), ISSN: 2319-5967, Volume 1, Issue 2, November ,2012,pp.246-254.

[7] Xu-Bao Shun and Shun-Shi Zhong, "An Adaptive Beamforming Approach Using Online Learning Neural Network", IEEE, School of Communication and Information Engineering ,Shanghai University, China , ISBN:0-7803-8302-8, June 2004 ,pp.2663-2666.

[8] Ahmed H. El Zooghby, , Christos G. Christodoulou, and Michael Georgiopoulos, "A Neural Network Based Smart Antenna for Multiple Source Tracking “, IEEE , TRANSACTIONS ON ANTENNAS AND PROPAGATION, Electrical and Computer Engineering Department, University of Central Florida, Orlando, FL 32816 USA., VOL8, NO. 5, May 2000,pp.768-775.

[9] Halil Yigit, Adnan Kavak and H .Metin Ertunc "Using Autoregressive and Adaline Neural Network Modeling to Improve Downlink Performance of Smart Antennas",IEEE, Department of Electronic and Computer Engineering, Kocaeli University, Izmit, Turkey, ISBN:0-7803-8599-3,June 2004,pp.165-170.

[10] Nuri Celik, , Wayne Kim, Mehmet F. Demirkol, Magdy F.Iskander, Rudy Emrick, "Implementation and Experimental Verification of Hybrid Smart-Antenna Beamforming Algorithm",IEEE, Hawaii Center for Advanced Communication, University. of Hawaii, Honolulu, HI, VOL. 5, April 2006,pp280-283.
[11] Ross D. Murch and Khaled Ben Letaief, "Antenna Systems for Broadband Wireless Access “,IEEE Communications Magazine, Hong Kong University of Science and Technology, Department of Electrical and Electronic Engineeringof Science and Technology, China, ISSN :0163-6804, April 2002,pp76-83.

[12] Heikki Koivo and Mohammed Elmusrati , "Smart Antennas",Systems Engineering in Wireless Communications, John Wiley and Sons, Ltd. ISBN: 9780-470-02178-1,2009, ,pp 261-302.

[13] Mohammad Tariqul Islam and zainol Abidin Abdul Rashid, "MI-NLMS Adaptive Beamforming Algorithm For Smart Antenna System Applications", Journal of Zhejiang University Science, Department of Electrical, Electronics and System Engineering, Faculty of Engineering, University of Kebangsaan Malaysia, ISSN 1009-3095,July 2006 pp.1709-1716 .

[14] Jian Li and Petre Stoica, "Robust Adaptive Beamforming “,J OHN WILEY \& SONS, INC, ISBN: 10 0-47167850-3,2005.

[15] D.M. Rodvold, D.G. McLeod, J.M. Brandt, P.B. Snow, and G.P. Murphy, "Introduction to Artificial Neural Networks for Physicians: Taking the Lid Off the Black Box“, Wiley-Liss,Inc., Volume 46, Issue 1, January 2001, pp.39-44.

[16] A. H. El Zooghby, M. Georgiopoulos and C. G. Christodoulou , "Neural Network-Based Adaptive Beamforming for One- and Two-Dimensional Antenna Arrays“, IEEE, Electrical and Computer Engineering Department, University of Central Florida, Orlando, FL 32816 USA, December 1998 pp.1891-1893.

[17] Laurene Fausett, " Fundamentals Of Neural Networks Architectures, Algorithms and Applications “,PrenticeHall,ISBN: 0133341860, 9780133341867,1994.

[18] Wai-Kai Chen, "Neural Networks and Computing Learning Algorithms and Applications", SERIES IN ELECTRICAL AND COMPUTER ENGINEERING, University of Illinois, Chicago, USA), ISBN-10 186094-758-1,2007.

[19] Medsker L.R. and L.C. Jain, "Recurrent Neural Networks Design And Application” ,CRC Press, Boca Raton London New York Washington, D.C, 2001.[48] Graves A., Hinton G, and Mohamed A. . "Speech Recognition with Deep Recurrent Neural Networks" Department of Computer Science, Toronto University, PP.1-5, 2012

[20] Ben Krose and Patrick van der Smagt,"An Introduction to Neural Networks", $8^{\text {th }}$ eddition, Amsterdam University, November 1996.

[21] Guez-Estrello , Carmen B. Rodr, and Felipe A. Cruz Pérez, "An Insight into the Use of Smart Antennas in Mobile Cellular Networks“, University Campus ,Electric Engineering Department, ISBN 978-953-307-2463,April 2011 